\title{
Edición de los escritos de juventud de Nietzsche: Las primeras notas de los fragmentos póstumos del filósofo. Un informe sobre su investigación
}

\author{
JOHANN FIGL
}

Los actos conmemorativos celebrados en el año 2000 con motivo del centenario de la muerte de Nietzsche (25 de agosto de 1900) han puesto claramente de relieve una vez más la importancia de este filósofo para el presente. El pensamiento de Nietzsche no solamente es válido como programa de un outsider, sino que ideas centrales de sus análisis críticos se han convertido, para muchas personas de nuestra cultura, en componentes de autocomprensión; esto es cierto tanto respecto a temas filosóficos y teórico-científicos como para cuestiones religiosas o psicológicas.

Ante el panorama del número no despreciable de publicaciones de Nietzsche y de la penetrante relevancia cultural de este pensador, va aumentando la importancia de la edición textualmente fidedigna e íntegra de sus escritos, que fue llevada a cabo por los estudiosos italianos Giorgio Colli y Mazzino Montinari y fue completada casi en su totalidad; desde 1967 se han publicado en la editorial Walter de Gruyter (Berlín/Nueva York) numerosos volúmenes de la edición crítica de las obras $^{1}$ y de las cartas ${ }^{2}$ de Friedrich Nietzsche.

1 F. Nietzsche, Werke. Kritische Gesamtausgabe, ed. G. Colli y M. Montinari, continuada por W. Müller-Lauter y K. Pestalozzi, Berlin/New York, W. de Gruyter, 1967 ss., alrededor de 40 tomos repartidos en 9 secciones (citaremos esta edición como KGW, indicando la sección en números romanos y el tomo en arábigo).

2 F. Nietzsche, Briefwechsel. Kritische Gesamtausgabe, ed. G. Colli y M. Montinari, continuada por N. Miller y A. Pieper, Berlin/New York, W. de Gruyter, 1975 ss., cerca de 22 tomos repartidos en 3 secciones (citado como KGB, indicando sección y tomo). 
Desgraciadamente Mazzino Montinari no pudo completar en su integridad la edición a causa de su muerte repentina en 1986; Giorgio Colli había muerto ya años antes. La continuación de la edición se encontró con grandes problemas: de las ocho secciones de la obra, proyectadas en un primer momento, y que luego fueron nueve, sólo habían aparecido completos los volúmenes de las secciones III-VIII; pero tan sólo una parte de los Nachbericht (los comentarios filológicos, dedicados a crítica de los textos, comentarios, descripción de los manuscritos, etc)3.

Todavía quedaban pendientes algunos tomos de la sección II (Philologica) y toda la sección I (Jugendschriften). Gracias a los esfuerzos de Wolfgang Müller-Lauter (Berlín) y Karl Pestalozzi (Basilea), se pudo continuar de inmediato la edición de Colli y Montinari; y ciertamente con el apoyo de la cooperación internacional. El autor de este informe fue invitado a hacerse cargo de la Edición de los Escritos de Juventud (Jugendscbriften); puesto que yo había estudiado en los años anteriores en el Archivo GoetheSchiller en Weimar, durante prolongadas estancias de investigación, los aspectos centrales de los legados póstumos de la infancia y juventud de Nietzsche, y pude convencerme de la importancia de las notas no publicadas del joven Nietzsche para muchos problemas, especialmente para los planteamientos filosófico-religiosos ${ }^{4}$, me di cuenta entonces que se trataba de una tarea relevante para distintas disciplinas científicas. Después de que hubiese sido atendida mi propuesta por la Österreichischen Forschungsförderungsfonds (FWF) y una solicitud correspondiente para un colaborador científico, acepté la dirección de este proyecto de edición. El Dr. Hans Gerald Hödl trabaja conmigo desde 1989 en la elaboración de los escritos de juventud; desde 1994 hasta el 2000 estuvo como colaborador científico el filólogo de Salzburgo Dr. Ingo W. Rath, especialmente para los póstumos de la época de estudiante de Nietzsche (1864-1869). Entretanto están ya listos en la imprenta tres volúmenes (1, 2 y 4 ) de los cinco volúmenes previstos 5 , los otros dos volúmenes pendientes, el 3 y el

3 Véase el Nachbericht a las secciones IV y VII, en las Kritische Studienausgabe (= KSA). Montinari, anticipándose, había comentado todos los escritos (excepto los juveniles y los filológicos): F. Nietzsche, Sämtliche Werke. Kritische Studienausgabe, 15 vols., ed. G. Colli y M. Montinari, vol. XIV: Comentario a los volúmenes 1-13, Berlin/München, W. de Gruyter / dtv, 19882.

4 Cf. J. Figl, Dialektik der Gewalt. Nietzsches hermeneutische Religionsphilosophie. Mit Berücksichtigung unveröffentlichter Manuskripte, Düsseldorf, 1984.

5 Véase un poco más adelante los volúmenes mencionados. 
5, aparecerán previsiblemente a finales del 2001 o principios del 2002, y después de ellos el volumen de los Nachbericht a la sección I (probablemente en dos tomos).

Por lo tanto, los escritos de juventud e infancia se han editado y elaborado de una manera nueva por un Grupo de trabajo austríaco.

El proyecto de edición de la sección I está vinculado a una cooperación internacional: en primera línea, respecto de los póstumos filológicos de la época de estudiante (volúmenes 4 y 5), existe aquí una estrecha colaboración con el profesor Glenn W. Most, del Seminar für Klassische Philologie de la Universidad de Heildelberg. Además hay una cooperación con los editores de los escritos de juventud en lengua italiana (profesor Carpitella, de la Universidad de Florencia, y el profesor Campioni, de la Universidad de Lecce), los cuales han editado a partir de nuestra edición (sección I, tomo 1), textos de la época de estudiante de bachiller ${ }^{6}$, así como con científicos que trabajan en el comentario de los volúmenes de cartas de la misma época (especialmente la Sra. Dra. Renate Müller-Buck, de Tübingen). Una serie de conferencias internacionales de los editores, en las que se habían interesado editores y redactores de distintas secciones — por ejemplo de los volúmenes de KGW y de KGB, de Alemania, Italia, Suiza (especialmente de la sección III, que contiene los escritos de la época de Basilea)—, garantizaron la coordinación de la edición completa de los escritos del filósofo.

\section{IMPORTANCIA Y NECESIDAD APREMIANTE DE UNA NUEVA EDICIÓN DE LOS ESCRITOS DE JUVENTUD DE NIETZSCHE ${ }^{7}$}

Los Schriften aus der Jugendzeit — dejando a parte los singulares informes de Elisabeth Förster-Nietzsche en las biografías sobre su hermano y la selección en el volumen 1 de la edición de Musarion ${ }^{8}$

6 Cf. F. Nietzsche, Scritti giovanili 1856-1864, en Opere, vol. I, tomo 1, a cura di Mario Carpitella, Milano, Adelphi, 1998, «Criteri dell’Edizione».

7 La siguientes explicaciones están tomadas en parte de mi contribución a la «Edition des Frühen Nachlasses Friedrich Nietzsches — grundsätzliche Perspektiven», en Nietzscheforschung. Eine Jabresschrift, I, 161-168; también están incluidas en parte en el Prólogo del mencionado volume

8 F. Nietzsche, Gesammelte Werke, Bd. 1: Jugendschriften. Dichtungen/Aufsätze/Vorträgel Aufzeichnungen und philologische Arbeiten 1858-1868, München, Musarion-Verlag, 1922, pp. $213-312$. 
fueron editados por primera vez en la Historisch-Kritischen Gesamtausgabe, que quedó incompleta, como volúmenes 1-5 de las obras9. En primer lugar hay que señalar que en ella los textos se han descifrado en gran medida de un modo filológicamente exacto; sin embargo, con motivo de mi dedicación a los originales en el Archivo Goethe-Schiller (GSA) me pude convencer ya en 1982, en una estancia de investigación, que frente a los hechos positivos mencionados había una grave carencia, que debería evitarse en todo caso en una nueva edición de los escritos de juventud: en primer lugar la BAW está incompleta; en segundo lugar no está uniformemente ordenada cronológicamente; y en tercer lugar tampoco está concebida sistemáticamente de modo satisfactorio.

Como carencia más grave debe aparecer el carácter incompleto de la BAW; en sentido estricto no es una edición completa, puesto que faltan grupos importantes de notas de Nietzsche, algo que por lo demás no está motivado en casos particulares. Se trata preferentemente de los siguientes grupos de textos:

a) Manuscritos que faltan en su totalidad:

- De la época de los estudios escolares: traducciones, copias, dictados, listados de libros, extractos, noticias escolares, etc. (véase por ejemplo BAW II 457-459, donde estos son simplemente mencionados).

- De la época de estudiante: especialmente todos los apuntes de clase (véase BAW I, LIV-LIX); además otras notas;

- Hay que añadir transcripciones de poesías, que son presentadas con ocasión del Año Nuevo o del cumpleaños de los más allegados, las cuales son fechadas sólo en parte según el motivo (por ejemplo, año nuevo de 1852; cumpleaños de la madre, 1852).

b) Partes de los manuscritos: numerosos apuntes explicativos, o que aparecen también como incidentalmente (por ejemplo, instructivas anotaciones), no son reproducidos — faltan variantes del texto y proyectos.

9 Editado por H. J. Mette y otros, München, C. H. Beck'sche Verlagsbuchhandlung, 1933-1940; designado con la sigla BAW. 
La cronología contradictoria se manifiesta ante todo en que BAW coloca los primeros apuntes del niño Nietzsche en el anexo al volumen 1 , mientras que el primer volumen habría de comenzar cronológicamente precisamente con ellos. También en los otros volúmenes se encuentran despropósitos, por ejemplo en el volumen 3, al interferir cronológicamente los «apuntes filosóficos» (p. 317 ss.) con los filológicos, que van inmediatamente antes. A esto se une una falta de sistematicidad en el planteamiento. Parece problemático extraer de los manuscritos determinadas notas que serían fácilmente reconocibles con facilidad «como una especie de cuerpo extraño puramente filosófico» (BWA volumen 3, Nachbericht p. 439). Además de esta problemática, hay que añadir otra general que recorre la edición completa de la BAW: el problema de cómo se han de incluir o excluir las apostillas no ha sido dilucidado en cada caso concreto. Para ello habría tenido que ser sistemáticamente aclarado, de qué modo se ha de juzgar en cada caso concreto las correspondencias con los Philologica, que en KGW aparecen en una sección propia (II).

Por estos motivos se publican por primera vez y completamente en la sección I de la edición crítica de la obra de Nietzsche (KGW I) las primeras notas de la infancia y juventud de Nietzsche. De la misma manera que con la edición crítica completa de las cartas (KGB I 1), la cual incluye cartas desde 1850, es decir, las primeras cartas de la infancia de Nietzsche, se publican también los restantes escritos póstumos de la infancia y juventud desde los más tempranos - comenzando con la nota de una poesía de Año Nuevo, que fue puesta por escrito hacia el año nuevo de 1852. A través de las notas y escritos contenidos en la primera sección se documenta ampliamente el camino del pensamiento, formación e instrucción del joven Nietzsche. Justamente en un filósofo, para el que las cuestiones de la relación entre la vida y el pensamiento, y sobre todo las de la genealogía y el devenir, debían alcanzar una importancia central, puede cobrar también una relevancia especial el interés por los comienzos de su propio camino biográfico y anímico. Pero también, independientemente de estos planteamientos especializados, las primeras notas de Nietzsche son de gran importancia sobre todo a causa de su valor histórico y cultural y de su especial relevancia histórico-filosófica: que yo sepa, no se conoce de ninguna otra personalidad relevante del siglo XIX, un legado póstumo de la infancia y juventud que se 
pueda comparar en extensión; en todo caso no están publicados. Partiendo de los motivos mencionados, se pretende una completud fundamental con la publicación de las primeras notas en la sección I.

Los estudios de archivo mostraron cuán extenso es el legado póstumo desconocido hasta ahora, por no estar editado, de la primera y más temprana época de Nietzsche; para el interés mismo de las investigaciones es necesaria una edición completa de las notas de la infancia, y no sólo por intereses filosóficos, o intereses biográficos generales, sino porque estos póstumos —en su abundancia y extensión singulares para uno de los grandes pensadores de la Modernidad- ofrece a numerosas disciplinas científicas un excepcional material de fuentes, que no se encuentra tan fácilmente: para psicólogos y pedagogos, para filólogos, germanistas e historiadores, para críticos de la cultura y en general para especialistas en ciencias humanas; para estudiosos de la religión y para teólogos.

Los criterios de edición seguidos en los volúmenes de la sección I concuerdan en general con los de la edición completa KGW; y ciertamente en sus tres principios: fidelidad a los manuscritos, orden cronológico y que sea una edición completa. Los requisitos específicos de la sección - en cuanto que en la sección I se da el hecho especial de las notas que recorren todo el Nachlass, procedentes de la fase de socialización escolar y están unidas de modo natural con muchos elementos receptivos-, había que tenerlos adicionalmente en cuenta.

\section{LAS PRIMERAS NOTAS DE LA INFANCIA Y LOS PRIMEROS AÑOS DE LA ESCUELA Y EL BACHILLERATO}

Tomo I 1: Apuntes póstumos: principios 1852 - verano 185810

A los textos escritos y compuestos por Nietzsche, a partir aproximadamente de 1854, pertenecen poesías, piezas de teatro, proyectos, etc., así como apuntes biográficos. Los llamados 'apuntes oca-

10 F. Nietzsche, Werke. Kritische Gesamtausgabe, Abteilung I, Band 1: Nachgelassene Aufzeichnungen: Anfang 1852 - Sommer 1858, realizado por J. Figl, con la colaboración de Hans Gerald Hödl, Berlin/New York, W. de Gruyter, 1995.

11 Cf. M. Montinari, Nachbericht, en KGW IV 4, p. 492; y KGW VII 4/2, p. 562. 
sionales'11 — como por ejemplo notas sobre precios, registro de un libro, noticias de tipo personal, y otros- son registrados si guardan relación en cuanto al contenido con los otros textos de este volumen; las restantes anotaciones de esta clase son notificadas en el Nachbericht. Muchas de las composiciones de Nietzsche van acompañadas con signos y esquemas gráficos; si ellas pertenecen inmediatamente al nexo textual, en cierta medida forman una parte constitutiva del mismo, entonces también son en esos casos reproducidos los signos (sigla Z) juntamente con los textos. Además de ello se encuentran en los póstumos signos que no están en un contexto textual mayor, y que por ello no se llevan al volumen del texto, sino al volumen del comentario (como facsímil).

Además de los textos mencionados, se conservan en el legado póstumo de la época escolar de Naumburg un número de notas que - como se ha mencionado- ciertamente no son composiciones concebidas personalmente, pero que no pueden considerarse sin importancia para la comprensión de su primer desarrollo. La edición quiere hacer justicia al carácter específico de estos materiales, que deriva de la constitución propia de un legado póstumo de la infancia o de la juventud, registrando tales textos en una parte complementaria (anexo). Son aquellos textos - comenzando con los de 1852 - cuya composición casi por regla general la había pretendido Nietzsche, o bien que fuera encargada, tanto por la familia (como una poesía festiva especialmente preformulada), como por la escuela (como material escolar, como por ejemplo escritos de dictado así como especialmente traducciones). A causa de este origen específico se trata con frecuencia de apostillas, que por ello son señaladas desde un principio con una sigla propia (A).

Estos textos ponen de relieve gráficamente los primeros pasos en la formación de Nietzsche, que para su posterior desarrollo han tenido su importancia; además estos manuscritos documentan un encuentro muy temprano de Nietzsche con contenidos determinados e ideas y otros numerosos aspectos importantes para la génesis de su obra y para su biografía; son también testimonios de especial valor histórico y cultural, incluso desde el punto de vista interdisciplinar (pedagógico, investigación de la historia escolar y del contenido de las enseñanzas, investigación biográfica y de la infancia, historia de la devoción cristiana, etc). 


\section{Fragmentos póstumos de la époCa del bachillerato en la Escuela de Pforta}

Tomo I 2: Apuntes póstumos: otoño 1858 - otoño 186212

Tomo I 3: Apuntes póstumos: otoño 1862 - verano 1864 (aparecerá en 2001/02)

En el primero de los dos volúmenes de la Escuela de Pforta están publicadas las notas de los primeros cuatro años que Nietzsche ha pasado en la escuela de élite de Pforta. El tomo 3, que se encuentra en preparación (Escuela de Pforta 1862-1864), aparecerá previsiblemente hacia el 2002.

Junto a trabajos compuestos más o menos de motu propio, como por ejemplo poesías o conferencias para la Asociación de amigos «Germania», poesías o notas personales, como diarios, existe — como en KGW I 1 - una parte importante del material presentado en el volumen I 2 a partir de trabajos en la escuela y para la escuela, lo que también se aplica a una parte de las poesías. En la publicación se ha hecho un esfuerzo por editar también en su integridad el copioso y variado material de apuntes manuscritos de Nietzsche en el ámbito escolar que ha llegado hasta nosotros; no obstante, una parte de ese material (como por ejemplo los de carácter puramente técnico-formal, como apuntes de asignaturas prácticas, de ejercicios, etc.) no está impresa en los tomos de texto, sino que ha sido reproducida en el Nachbericht. En el volumen en cuestión se recogen — de manera análoga al tomo I 1 - con la sigla A los extractos o resúmenes conservados de Nietzsche (por regla general de obras históricas o histórico-literarias). Estos textos impresos en un pequeño escrito contienen ese material, el cual, aunque son extractos, remiten de manera muy clara a la ocupación personal de Nietzsche. Esto se ve con claridad, por ejemplo, en los extractos de la Historia de los últimos 40 años de Menzel o de la Historia de la sociedad de Mundt, de cuyo libro se conserva también una reflexión personal de Nietzsche de la misma época. Los otros extractos reproducen también lo que Nietzsche ha asimilado de una manera en parte muy individual. Por lo demás, el

12 F. Nietzsche, Werke. Kritische Gesamtausgabe, Abteilung I, Band 2: Nachgelassene Aufzeichnungen: Herbst 1858 - Herbst 1862, editado por J. Figl, preparado por Hans Gerald Hödl, con la colaboración de Ingo Rath, Berlin/New York, W. de Gruyter, 2000. 
trabajo de resumir a menudo va más allá de la mera recepción hacia una propia configuración del texto.

Muchos de los textos reproducidos en este volumen como apuntes personales son del mismo tipo, así una nota, que se incluye como una breve reflexión independiente en un dictado procedente de la clase de religión sobre el Concilio de Nicea y que comenta una sentencia de Arrio, o también los artículos de latín que tratan temas históricos.

Algunos textos no tenidos en cuenta en las ediciones que se han publicado hasta ahora de los escritos juveniles de Nietzsche han podido ser identificados en base a las informaciones escolares, como notas en relación con tareas puestas. Para fecharlos se ha tenido que recurrir en algunos casos tanto a criterios internos (como por ejemplo una cierta soltura en el lenguaje) como al criterio externo del desarrollo del manuscrito.

Se ha buscado sobre todo la estricta reproducción del soporte transmitido, pero esto podía no alcanzarse en todos los casos, por ejemplo cuando Nietzsche redactó un manuscrito con anotaciones del tipo más diverso sin ninguna sucesión ordenada; los textos de un manuscrito semejante han sido ordenados según su pertenencia cronológica a los grupos de texto o grupos de fragmentos correspondientes.

\section{Fragmentos póstumos de La época de eStudiante (A PARTIR DEL OTOÑO 1864)}

Tomo I 4: Apuntes póstumos: otoño 1864 - primavera 186813

Tomo I 5: en fase de elaboración

De los dos tomos previstos para la época de estudiante en la sección I (I 4 y I 5) ha aparecido el primero en 1999.

Las notas a partir del otoño 1864 hasta comienzos de la época de Basilea (1869) comprenden una gran cantidad de apuntes, proyectos y esbozos, así como también de secciones independientes en sí mismas. Este material manuscrito, que comprende varios miles de páginas, pertenece a ámbitos temáticos diferentes: casi la mitad de las

13 F. Nietzsche, Werke. Kritische Gesamtausgabe, Abteilung I, Band 4: Nachgelassene Aufzeichnungen: Herbst 1864 - Frïhjahr 1868, ed. J. Figl, preparado por W.'Rath, Berlin/ New York, W. de Gruyter, 1999. 
notas son de contenido biográfico, mezcladas con las de contenido filosófico y filológico-filosófico, la parte restante del material es de contenido filológico. La edición tiene que hacer justicia a este carácter complejo y heterogéneo de la notas de la época de estudiante.

Para solucionar esta tarea adecuadamente, en la publicación de los materiales póstumos de la época a partir del otoño de 1864 se ha procedido del siguiente modo: aquellas notas (trabajos preparatorios, etc.) que ya fueron agrupadas en torno a los escritos contenidos en la segunda sección (Philologica), así como trabajos adicionales de pura filología textual, han sido recogidas en todo caso allí, mientras que el resto completo de los póstumos de la época de estudiante ha sido publicado de forma fiel en la sección $I$.

El tomo 4 en cuestión documenta así también la evolución de Nietzsche en su confrontación con los planteamientos filológicos.

A diferencia de la edición precedente de estos escritos por parte de H. J. Mette y K. Schlechta (BAW 3-5), los cuales practicaron una separación parcial de apuntes filológicos y filosóficos, aquí se prescinde de una diferenciación estricta semejante. En las observaciones previas de los editores de la nueva edición de la BAW, escribe Rüdiger Schmidt con razón: «Según el estado actual de la investigación no se puede ya justificar una drástica separación de los textos filológicos y filosóficos. El intento de Schlechta, de practicar una tal separación apartándose de los principios de edición originales en BAW III, ya no parece que hoy tenga sentido»14. La sección I de la KGW tiene en cuenta esta crítica, con las limitaciones que acabamos de mencionar y justificar.

La exactitud filológica en el desciframiento así como la integridad disminuyen algo en la BAW (desde el volumen 3 y siguientes) para la época de estudiante frente al volumen 1 y 2 . El estudio atento del material original de los manuscritos de Nietzsche en el Archivo de Goethe-Schiller en Weimar mostró además que incluso una serie de manuscritos fue dejada al margen sin ninguna razón. En este tomo se publican completamente por primera vez los póstumos de la época de estudiante, tal y como está previsto para la sección I. Además se incluyen anotaciones del tipo más diverso, que aquí en parte han sido

14 Reimpresión fotomecánica bajo el título Frühe Schriften, con un prólogo de R.Schmidt, 5 Bde., München, C.H. Beck, 1994. 
publicadas por primera vez, como por ejemplo apuntes autobiográficos, fragmentos líricos, poesías de broma, apuntes filosóficos, científicoreligiosos y teológicos.

Este cuarto tomo de la sección I comprende así —prescindiendo de los apuntes mencionados, pertenecientes a la sección II — las notas de Nietzsche que van desde el otoño de 1864 hasta la primavera de 1868, por lo tanto la época de sus años de estudiante en Bonn, los dos años siguientes de estudiante en Leipzig y la época de su servicio militar, hasta poco después de su accidente ecuestre en marzo de 1868. El fundamento de todos los textos impresos es — como en todos los textos de la edición - el material original manuscrito en el Archivo de Goethe-Schiller en Weimar.

\section{CONTINUACIÓN DE LA PLANIFICACIÓN EN TORNO AL NACHBERICHT DE LA SECCIÓN I}

En breve estarán listos todos los tomos de texto de los escritos juveniles de Nietzsche. Un trabajo sucesivo formaría el volumen de Nachbericht para la sección I, que presumiblemente aparecerá en dos tomos - uno para la época escolar y otro para la época de estudiante. Estos tomos tendrán como contenido, además de un compendio biográfico, la crítica textual, el comentario y la descripción del manuscrito, así como un índice detallado. Además de eso serán publicados en conexión con el comentario filológico los materiales de la época escolar, que son primariamente de tipo técnico y formal (como ejercicios de ortografía y de estilo, así como otros materiales de ejercicios; listas de vocablos, etc). Una tarea especial respecto a la época de estudiante la conforman los apuntes de clase, la mayor parte dictados de las clases de filología (cerca de 1100 páginas de manuscritos), a las que Nietzsche ha asistido; en mucho menor volumen (cerca de 150 páginas de manuscrito) de clases de Teología, Historia de la Filosofía e Historia del Arte (de la época de Bonn); de las clases de Historia de la Literatura Alemana, de Arqueología15 y de una Introducción a la

15 Sobre estos apuntes de clase véase H. Cancik, Otto Jahns Vorlesung «Grundzüge der Archäologie», Bonn, Sommer, 1865, en las anotaciones de Eduard Hiller y Friedrich Nietzsche, en W. M. Calder III, H. Cancik, B. Kytzler (ed), Otto Jahn (1813-1868). Ein Geisteswissenschaftler zwischen Klassizismus und Historismus, Stuttgart, 1991, pp. 29-56. 
Ciencia Política y Jurídica (Universidad de Leipzig) Debido al gran volumen de apostillas filológicas de las clases, se producen problemas adicionales en relación al tipo y extensión de su publicación. A causa de la importancia que tienen las apostillas de clase de Nietzsche respecto a su proceso de formación, como, por ejemplo, puede ser dicho sin reserva respecto a las apostillas de clase de teología y filosofía ${ }^{16}$, está previsto publicar en imprenta las apostillas de clase no filológicas.

\section{Primeras notas de Nietzsche — un proyecto de edición AUSTRÍACO (OBSERVACIONES FINALES)}

Los volúmenes descritos son un proyecto de trabajo, con cargo a los Fondos Austríacos para la Demanda de Investigación Científica (FWF) y llevado a cabo bajo la dirección o colaboración de científicos austríacos.

De esta manera se ha producido una especial relación entre la obra de Nietzsche y Austria, después de haberse dado ya en vida del filósofo una primera estrecha relación: aquí tuvo él su primer círculo de admiradores entre los estudiantes de la Universidad de Viena, el Círculo de Lectores de Estudiantes Alemanes, al que también pertenecía Freud ${ }^{17}$. En el terreno editorial fue significativa en otro contexto la incidencia de un científico originario de Austria: es sabido que Karl Schlechta, vienés de nacimiento, descubrió las manipulaciones de la hermana de Nietzsche, Elisabeth Förster-Nietzsche, con una 'obra' bajo el título de La voluntad de poder, e hizo posible que se publicaran por primera vez de un modo auténtico los apuntes tardíos póstumos ${ }^{18}$; en el proyecto mencionado se trata de los primeros, mejor

16 Cf. sobre ello J. Figl, Dialektik der Gewalt. Nietzsches hermeneutische Religionsphilosophie, Düsseldorf, 1984; y «Nietzsches frühe Begegnung mit dem Denken Indiens. Auf der Grundlage seiner unveröffentlichten Kollegnachschrift aus Philosophiegeschichte (1865)», Nietzsche-Studien, 18 (1989), 455-471.

17 Sobre esto véase Johann Figl, «Nietzsche und Wien», en Von Nietzsche zu Freud. Übereinstimmungen und Differenzen von Denkmotiven, Wien, 1996, 11-17; Hans Gerald Hödl, «Nietzsche in Österreich. Prometheische Religion: Siegfried Lipiners poetische NietzscheRezeption», en M. Benedikt - E. Kiss - R. Knoll (edts); H. Kohlenberger - J. Rupitz - W. Seitter (coedts), Verdrängter Humanismus - Verzögerte Aufklärung IV. Anspruch und Echo. Sezession und Aufbrüche in den Kronländern zum Fin-de-siécle. Philosopbie in Österreich (1880-1920), KlausenLeopoldsdorf/Klausenburg, Cluj-Napoca, 1998, pp. 379-396.

18 Werke in drei Bänden, ed. K. Schlechta, München, Hanser, 1954ss., 3 tomos y 1 tomo de índice. 
dicho primerísimos póstumos de Nietzsche, que ahora por vez primera serán publicados en su integridad con la aparición de los volúmenes restantes de la Sección I. Mientras que la 'desconstrucción' de La voluntad de poder ha incidido esencialmente en la historia de la recepción y de sus efectos, en los escritos de juventud se trata de su propia recepción de textos, motivos, etc., previamente dados, de la primera formación de su propia filosofía en un encuentro con una profusión de contenidos, que le pueden haber influido o bien de los que él se liberó. En cierta medida se trata de la 'génesis' de su pensamiento, que puede ser reseñada y analizada documentalmente desde las primeras formas de expresión. El interés científico se vuelve al niño y al joven, por lo tanto al Nietzsche que escribe y piensa en una fase biográficamente temprana de su desarrollo.

Una decisión filosófica y antropológica previa es qué relevancia se le atribuye a esta fase 'genética' de la vida — no sólo respecto a la obra posterior, sino la que le corresponde en sí, aunque también tiene una relevancia especial la cuestión de la relación de estos primeros apuntes con la obra del Nietzsche maduro. Como editor de los escritos de juventud de Nietzsche estoy convencido de la gran relevancia de estas notas para la comprensión del conjunto del pensamiento de Nietzsche - en especial también para su evolución religiosa. Ciertamente esto es una valoración al margen del trabajo en la edición, que puede ser corroborada en estudios particulares. Respecto a la edición es necesario poner de una manera apropiada a disposición de la investigación ${ }^{19}$ este texto como tal. Sólo sobre esta base pueden ser hechas de una manera fundada afirmaciones de gran alcance sobre la importancia, para su obra madura, del desarrollo espiritual del primer Nietzsche.

traducción: Luis E. de Santiago Guervós

19 Cf. por ejemplo T. H. Brobjer, «Nietzsche's Education at the Naumburger Domgymnasium 1855-1858», Nietzsche-Studien, 28 (1999), 302-322, el cual por primera vez aprovecha científicamente materiales publicados en KGW I 1 de la época de Naumburg. 